\title{
Ein Vergleich randomisierter Antworttechniken bei Ziehen ohne Zurücklegen
}

\author{
Andreas Quatember und Christoph Freudenthaler \\ IFAS-Institut für Angewandte Statistik, Linz
}

\begin{abstract}
Zusammenfassung: Wird bei ,heiklen“ Themen bei direkter Befragung eine hohe Nonresponse- bzw. Falschantwortrate riskiert, dann bieten randomisierte Antworttechniken eine alternative Möglichkeit zur Erzielung unverzerrter Schätzer für interessierende Parameter. Man bezahlt dafür mit einer Erhöhung der Ungenauigkeit der Schätzer. In diesem Aufsatz werden für zwei solche Schätzer für Anteile ihre Varianzen bei Ziehen ohne Zurücklegen hergeleitet. Ferner werden die Stichprobenverteilungen weiterer mit solchen Befragungsdesigns erhobener Schätzer in einer Simulationsstudie miteinander verglichen und grafisch veranschaulicht. Die Ergebnisse geben Auskunft über die Qualität von insgesamt 24 Schätzern, ihre Unterschiede und Grenzen.
\end{abstract}

\begin{abstract}
With sensitive subjects there is the risc of a high nonresponse- or untruthful answers rate with direct questioning on this matter. In this case randomized response techniques are an alternative for an unbiased estimation of the parameters under study. The price that has to be paid for this is an increase of the variance of the estimators. In this paper for two of such estimators for proportions the variances are derived for simple random sampling without replacement. Furthermore by a simulation study the sampling distribution of various estimators, that are based on such questioning designs are compared and graphically presented. The results give information about the quality of altogether 24 estimators, their differences and limitations.
\end{abstract}

Keywords: Datenqualität, Stichprobenerhebung, Randomisierte Antworttechniken, Nonresponse, Ziehen ohne Zurücklegen, Effizienz.

\section{Einleitung}

Der Bereich der statistischen Stichprobentheorie setzt sich mit den Auswirkungen unterschiedlicher Stichprobenverfahren und verschiedener Schätzmethoden auf die Qualität von aus Stichproben gewonnenen Schätzern für unbekannte Parameter auseinander. Sie ist eine reine Fullresponsetheorie, die in ihrem Urnenmodell den Fall nicht vorsieht, dass ausgewählte Kugeln sich nicht in der Urne befinden oder uns die Auskunft über ihre Farbe verweigern. Mit diesem Problemfeld beschäftigt sich die Theorie der fehlenden Werte, deren prominenteste Protagonisten die Amerikaner Roderick J.A. Little und Donald B. Rubin sind. Ein Ziel des am IFAS - Institut für Angewandte Statistik der Johannes Kepler Universität Linz eingerichteten institutsinternen Forschungsprojekts „Datenqualität in Stichprobenerhebungen“ ist es, ein in der Praxis anwendbares Manual zu erstellen, das diese beiden Theorien miteinander verknüpft (für nähere Informationen zu diesem Forschungsprojekt siehe in der Forschungsdokumentation der Johannes Kepler Universität Linz: https://fodok.jku.at/fodok/forschungsprojekt.xsql?FP_=1422). 
Das Auftreten von Antwortausfällen, also von „Nonresponse“, in Stichprobenerhebungen kann sich sowohl in einer verzerrten Schätzung von Parametern als auch in einer Erhöhung der Ungenauigkeit der erhobenen Schätzer niederschlagen. Bevor statistische Methoden wie die Gewichtsanpassung und die Imputation zur Kompensation aufgetretenen Nonresponses eingesetzt werden, ist natürlich zuallererst dafür Sorge zu tragen, diesen so gering wie möglich zu halten. Denn selbst im besten Fall lässt sich mit diesen Verfahren zumindest eine Erhöhung der Varianz des Schätzers im Vergleich zum Fullresponsefall nicht vermeiden.

Sofern eine Ursache für den Antwortausfall die Verweigerung der Beantwortung auf Grund einer ,heiklen“ Thematik (wie Drogenmissbrauch, Sexualverhalten, Abtreibungen oder kriminelle Delikte) ist, kann die Verwendung von für solche Fälle entwickelten randomisierten Antworttechniken dazu beitragen, die Verweigerungsrate zu minimieren. Den Befragungsstrategien, die unter diesem Begriff subsumiert werden, ist gemeinsam, dass es dem Interviewer durch Anwendung eines vorgegebenen Befragungsdesigns unmöglich gemacht wird, die konkrete Frage, auf die der Respondent tatsächlich antwortet, zu identifizieren. Auf diese Weise kann der Interviewer aus der gegebenen Antwort nicht auf das Vorliegen bzw. Nichtvorliegen der heiklen Eigenschaft schließen. Die Idee dabei ist, dass dadurch dem Interviewten die Angst vor einem ihm unangenehmen „Outing“ gegenüber dem Interviewer so weit genommen werden kann, dass er - unabhängig davon, welche Frage er tatsächlich zur Beantwortung erhält - diese wahrheitsgetreu beantwortet bzw. die Antwort darauf nicht überhaupt verweigert.

\section{Methodenüberblick}

\subsection{Die grundlegenden Verfahren}

Als die Pionierarbeit auf dem Gebiet der randomisierten Antworttechniken darf Warners Aufsatz aus dem Jahre 1965 betrachtet werden. Seien $U$ die Grundgesamtheit von $N$ Erhebungseinheiten, $U_{A}$ die Menge jener $N_{A}$ Elemente aus $U$, die eine sensitive Eigenschaft $A$ aufweisen und $U_{A^{c}}$ die Menge der $N_{A^{c}}$-elementigen Gruppe, die diese nicht aufweisen $\left(N=N_{A}+N_{A^{c}}, U=U_{A} \cup U_{A^{c}}\right)$. Der interessierende Parameter $\pi_{A}$ sei die relative Größe von $U_{A}$

$$
\pi_{A}=\frac{N_{A}}{N} .
$$

Warners Befragungsdesign lässt sich dadurch charakterisieren, dass der Interviewte (z.B. durch zufällige Ziehung einer Karte) entweder mit Wahrscheinlichkeit $p$ die Frage „Gehören Sie zur Gruppe $U_{A}$ ?“ oder mit Wahrscheinlichkeit $1-p$ die alternative Frage „Gehören Sie zur Gruppe $U_{A^{C}}$ ?“ zu beantworten hat. Sei $\pi_{A}^{(i)}$ der Anteil der Träger der Eigenschaft $A$ in der „Urne“ zum Zeitpunkt der Befragung der $i$-ten Erhebungseinheit in der Stichprobe $s$, so ist die Wahrscheinlichkeit $\pi_{y}^{(i)}$ für eine ,ja-Antwort“ dieses Respondenten $i$ gegeben durch

$$
\pi_{y}^{(i)}=p \pi_{A}^{(i)}+(1-p)\left(1-\pi_{A}^{(i)}\right) .
$$

Bei Ziehen der Erhebungseinheiten uneingeschränkt zufällig mit Zurücklegen aus $U$ ist $\pi_{A}^{(i)}=\pi_{A} \forall i \in s$ und mit $\hat{\pi}_{y}$, dem Anteil der ,ja-Antworten“ in der Stichprobe, ist 
folglich

$$
\hat{\pi}_{A}^{W}=\frac{\hat{\pi}_{y}+p-1}{2 p-1}
$$

(mit $p \neq 0.5$ ) unverzerrter Schätzer für $\pi_{A}$ (1) (vgl. Warner, 1965, S.65). Da bei Ziehen ohne Zurücklegen gilt: $\mathrm{E}\left(\pi_{A}^{(i)}\right)=\pi_{A}, \forall i \in s$, gilt diese Schätzereigenschaft von $\hat{\pi}_{A}^{W}$ auch bei diesem Stichprobenverfahren.

Die Varianz von $\hat{\pi}_{A}^{W}$ ist unabhängig vom verwendeten Stichprobenverfahren eine Funktion der Varianz des Anteils an ,ja-Antworten“ in der Stichprobe. Bezeichnen wir mit $y_{i}$ die Antwort der $i$-ten Erhebungseinheit und ist

$$
y_{i}=\left\{\begin{array}{l}
1 \text { wenn } i \text { mit }, \text { ja“ antwortet } \\
0 \text { sonst. }
\end{array}\right.
$$

Dann gilt mit $\widehat{\pi}_{y}=\sum_{s} y_{i} / n$

$$
\begin{aligned}
V\left(\hat{\pi}_{A}^{W}\right) & =\frac{1}{n^{2}(2 p-1)^{2}} V\left(\sum_{s} y_{i}\right) \\
& =\frac{1}{n^{2}(2 p-1)^{2}}\left[\mathrm{E}\left(\sum_{s} y_{i}^{2}\right)+\mathrm{E}\left(\sum_{s(i \neq j)} y_{i} y_{j}\right)-\mathrm{E}^{2}\left(\sum_{s} y_{i}\right)\right] .
\end{aligned}
$$

Es ist der Wert des Summanden in der Mitte der eckigen Klammer von (2), der sich bei uneingeschränkter Zufallsauswahl ohne Zurücklegen von jenem bei Ziehen mit Zurücklegen unterscheidet. Denn bei Ziehen ohne Zurücklegen bleiben die Wahrscheinlichkeiten dafür, dass sich ein Respondent in $U_{A}$ befindet, im Gegensatz zu Ziehen mit Zurücklegen nicht konstant. Dies führt zu einer deutlichen Erhöhung des Aufwands bei der Herleitung der Schätzervarianz.

Gemeinsam mit der ebenso erhöhten Komplexität in ihrer formalen Darstellung liegen somit die Gründe dafür vor, dass in nahezu allen Veröffentlichungen über randomisierte Antworttechniken ausschließlich Ziehen mit Zurücklegen betrachtet wird. In kleinen Grundgesamtheiten (wie Betrieben, in denen z.B. der Anteil an Beschäftigten geschätzt werden soll, die Büromaterial entwenden) führt eine Verwendung des so berechneten Varianzschätzers aber bei tatsächlichem Ziehen der Personen ohne Zurücklegen zu einer Überschätzung der Ungenauigkeit von Schätzern wie $\hat{\pi}_{A}^{W}$, die sich in Form zu breiter approximativer Konfidenzintervalle manifestiert.

Die Schätzervarianz (2) ist bei Warners Technik bei uneingeschränkter Zufallsauswahl von $n$ Elementen und Ziehen mit Zurücklegen (bzw. bei Ziehen ohne Zurücklegen aus einer großen Grundgesamtheit annähernd)

$$
V\left(\hat{\pi}_{A}^{W}\right)=\frac{\pi_{A}\left(1-\pi_{A}\right)}{n}+\frac{p(1-p)}{n(2 p-1)^{2}}
$$

(siehe Warner, 1965, S.65).

Nach einiger Rechnung gilt dafür bei Ziehen ohne Zurücklegen (vgl. J.-I. Kim und Flueck, 1978, S.347)

$$
V\left(\hat{\pi}_{A}^{W}\right)=\frac{\pi_{A}\left(1-\pi_{A}\right)}{n} \frac{N-n}{N-1}+\frac{p(1-p)}{n(2 p-1)^{2}} .
$$


Zur unverzerrten Schätzung von $V\left(\hat{\pi}_{A}^{W}\right)$ ist in (3) bzw. (4) $\pi_{A}$ durch $\hat{\pi}_{A}^{W}$ und im Nenner des ersten Summanden $n$ durch $n-1$ zu ersetzen. Der jeweils rechte der beiden Summanden in (3) und (4) ist die Varianzzunahme, die durch Warners Befragungsdesign im Vergleich zur direkten Befragung verursacht wird.

Die Varianzen $V\left(\hat{\pi}_{A}^{W}\right)$ sind umso größer, je näher sich die Wahrscheinlichkeit $p$ an 0.5 befindet. Gleichzeitig jedoch garantiert gerade eine solche Wahrscheinlichkeit ein erhöhtes Maß an Vertrauen in die Wahrung der Privatsphäre des Respondierenden. Diesen gegenpoligen Interessen muss bei der Wahl von $p$ Rechnung getragen werden. Dieser Designparameter sollte demnach idealerweise so festgelegt werden, dass er gerade so weit von 0.5 entfernt ist, dass noch die volle Kooperationsbereitschaft gewährleistet wird (siehe Greenberg et al., 1969, S.526).

Ist $p=1$ (bzw. $p=0$ ), so wird direkt nach der Zugehörigkeit zu $U_{A}$ gefragt. Die direkte Befragung ist somit jener Sonderfall von Warners Befragungsdesign, der die maximale Respondentenbelastung durch den geringsten Schutz der Privatsphäre mit der daraus resultierenden größten Nonresponse- bzw. Falschantwortwahrscheinlichkeit darstellt.

Greenberg et al. (1969) führten eine Idee, die Horvitz et al. (1967) präsentierten, theoretisch aus und trugen damit das zweite grundlegende Verfahren der randomisierten Antworttechniken bei. Ihr Befragungsdesign unterschied sich von jenem Warners durch das Ersetzen der alternativen Frage nach der Zugehörigkeit zur Menge $U_{A^{c}}$ durch die Frage nach der (mit der Zugehörigkeit zu $U_{A}$ nicht zusammenhängenden) Zugehörigkeit zu einer Gruppe $U_{B}$ der Größe $N_{B}$. Elemente der Menge $U_{B}$ sollen sich als Träger einer Eigenschaft $B$ auszeichnen, die als unverfänglich empfunden wird (z.B. der Geburtsmonat $B$ oder das Wohnbundesland $B$ ). Mit $\pi_{B}=N_{B} / N$ ist

$$
\hat{\pi}_{A}^{G}=\frac{\hat{\pi}_{y}-\pi_{B}(1-p)}{p}
$$

unverzerrter Schätzer von (1). Die Varianz des Schätzers (5) ist bei Ziehen mit Zurücklegen gegeben durch

$$
V\left(\hat{\pi}_{A}^{G}\right)=\frac{\pi_{y}\left(1-\pi_{y}\right)}{n p^{2}}
$$

(vgl. Greenberg et al., 1969, S.533). Diese Varianz lässt sich durch Ersetzen von $\pi_{y}$, der Wahrscheinlichkeit für eine ,ja-Antwort“, durch $\hat{\pi}_{y}$ und $n$ durch $n-1$ erwartungstreu schätzen. Bei Ziehen ohne Zurücklegen gilt

$$
V\left(\hat{\pi}_{A}^{G}\right)=\frac{\pi_{y}\left(1-\pi_{y}\right)}{n p^{2}}-\frac{n-1}{n(N-1)}\left[\pi_{A}\left(1-\pi_{A}\right)+\left(\frac{1-p}{p}\right)^{2} \pi_{B}\left(1-\pi_{B}\right)\right] .
$$

Der Beweis dafür befindet sich im Anhang. Der (positive) Subtrahend auf der rechten Seite von (7) ist der Effizienzgewinn durch Ziehen der Erhebungseinheiten ohne Zurücklegen im Vergleich zur mit Zurücklegen durchgeführten Ziehung. In großen Grundgesamtheiten gilt $(6) \approx(7)$.

Ist $\pi_{B}$ unbekannt, so ist für dieses Verfahren eine modifizierte Vorgehensweise zu wählen, um auch diese Variable schätzen zu können (siehe ebd., S.523ff). Hierbei wird die oben geschilderte Strategie in zwei unabhängigen Stichproben $s_{1}$ und $s_{2}$ mit Umfängen $n_{1}$ und $n_{2}\left(n_{1}+n_{2}=n\right)$ angewandt. Die Wahrscheinlichkeiten in den beiden Stichproben 
für die Frage nach der Zugehörigkeit zu $U_{A}$ werden mit $p_{1}$ und $p_{2}$ bezeichnet und es hat $p_{1} \neq p_{2}$ zu gelten. Der daraus resultierende erwartungstreue Schätzer $\hat{\pi}_{A}^{G^{\prime}}$ für $\pi_{A}$ ist (siehe ebd., S.525)

$$
\hat{\pi}_{A}^{G^{\prime}}=\frac{\hat{\pi}_{y_{1}}\left(1-p_{2}\right)-\hat{\pi}_{y_{2}}\left(1-p_{1}\right)}{p_{1}-p_{2}} .
$$

Greenberg et al. (1969) entwickelten die Varianz des Schätzers (8) bei Ziehen mit Zurücklegen (siehe ebd., S.525). Die überaus komplexe Varianzformel für Ziehen ohne Zurücklegen lässt sich bei J.-I. Kim and Flueck (1978) nachschlagen (ebd., S.348). Diese Varianzen hängen wesentlich von der Wahl der Wahrscheinlichkeiten $p_{1}$ und $p_{2}$ für das Stellen der heiklen Frage, der Stichprobenumfänge $n_{1}$ und $n_{2}$ und des Anteils $\pi_{B}$ ab. Greenberg et al. (1969) wiesen nach, dass $p_{1}$ und $p_{2}$ so weit wie möglich auseinanderliegen sollen und sie schlugen als Faustregel $p_{1}+p_{2}=1$ vor. Ferner soll hinsichtlich der optimalen Allokation des Gesamtstichprobenumfangs $n$ auf die beiden Stichproben $s_{1}$ und $s_{2}$ gelten (vgl. ebd., S.528)

$$
\frac{n_{1}}{n_{2}}=\sqrt{\frac{\pi_{y_{1}}\left(1-\pi_{y_{1}}\right)\left(1-p_{2}\right)^{2}}{\pi_{y_{2}}\left(1-\pi_{y_{2}}\right)\left(1-p_{1}\right)^{2}}} .
$$

Da die Wahrscheinlichkeiten $\pi_{y_{1}}$ und $\pi_{y_{2}}$ für eine ,ja-Antwort“ in $s_{1}$ bzw. $s_{2}$ auch von $\pi_{A}$ und $\pi_{B}$ abhängen, müssen diese zu diesem Zweck (z.B. aus früheren Erhebungen) zumindest grob geschätzt werden. Der größere Teil von $n$ wird dann jener Stichprobe zugeteilt, für die $p_{i}$ größer gewählt wurde $(i=1,2)$. Das Merkmal $B$ wiederum sollte danach ausgewählt werden, dass der Anteil $\pi_{B}$ auf derselben Seite von 0.5 wie $\pi_{A}$ so weit wie möglich von 0.5 entfernt ist (siehe ebd., S.526ff). Moors (1971) ergänzte die Überlegungen Greenberg et al.'s zur Festlegung der Wahrscheinlichkeiten $p_{1}$ und $p_{2}$ dahingehend, dass für $p_{1}>0.5$ konsequenterweise die Wahrscheinlichkeit $p_{2}=0$ gewählt werden und die 2. Stichprobe somit zur Gänze der Schätzung des Parameters $\pi_{B}$ dienen sollte.

\subsection{Modifikationen der grundlegenden Verfahren}

Warners und Greenberg et al.'s Befragungsdesigns bildeten die Basis für Weiterentwicklungen im Bereich randomisierter Antworttechniken. Eine Gruppe solcher Entwicklungen, die wir für unseren Vergleich unterschiedlicher solcher Verfahren heranziehen wollen, bilden zweistufige Befragungsdesigns. Diese sind dadurch charakterisierbar, dass sie vor Warners bzw. Greenberg et al.'s Methoden eine zusätzliche Randomisierungsstufe einschieben. Mangat and Singh (1990) etwa stellen in der 1. Stufe mit Wahrscheinlichkeit $q$ die Frage „Gehören Sie zur Gruppe $U_{A}$ ?“", während der Respondent mit der Gegenwahrscheinlichkeit $1-q$ zu Warners Vorgehensweise mit dem Designparameter $p$ verwiesen wird. Der erwartungstreue Schätzer $\widehat{\pi}_{A}^{M S}$ für $\pi_{A}$ ist gegeben durch (siehe ebd., S.440)

$$
\hat{\pi}_{A}^{M S}=\frac{\hat{\pi}_{y}-(1-q)(1-p)}{p+(2 q-1)(1-p)} .
$$

In einem weiteren zweistufigen Befragungsdesign (Mangat, 1992) wird in der 1. Stufe mit Wahrscheinlichkeit $q$ die Frage „Gehören Sie zur Gruppe $U_{A}$ ?" gezogen, während 
mit Wahrscheinlichkeit $1-q$ auf Greenberg et al.'s Design bei bekanntem $\pi_{B}$ verwiesen wird. Der unverzerrte Schätzer $\widehat{\pi}_{A}^{M 1}$ für $\pi_{A}$ zu diesem Befragungsdesign ist mit dem Designparameter $p$ gegeben durch (siehe ebd., S.84)

$$
\hat{\pi}_{A}^{M 1}=\frac{\hat{\pi}_{y}-(1-q)(1-p) \pi_{B}}{p+q(1-p)} .
$$

Die Varianzen der Anteilschätzer $\hat{\pi}_{A}^{M S}$ und $\hat{\pi}_{A}^{M 1}$ ergeben sich aus den Varianzen der Schätzer $\hat{\pi}_{A}^{W}$ und $\hat{\pi}_{A}^{G}$, wenn darin die Wahrscheinlichkeit $p$ durch $q+(1-q) p$ ersetzt wird. Die Vergleichbarkeit dieser Varianzen ist natürlich nur solange gegeben, solange die Befragten durch die zweistufige „Verschleierung“ dieser Erhöhung der Wahrscheinlichkeit für die Frage nach $U_{A}$ tatsächlich keinen Verlust an Privatsphäre empfinden und sich deshalb kooperationswillig zeigen. Auch die Wahrscheinlichkeit $q+(1-q) p$ darf demnach nicht beliebig gegen eins gehen.

Eine zweite Gruppe von Antworttechniken, die sich aus den Basisstrategien entwickelt hat, ist jene geschichteter Befragungsdesigns. J. M. Kim and Warde (2004) betrachten die Anwendung Warners Befragungstechnik in proportional bzw. optimal geschichteten Zufallsstichproben bei Ziehen mit Zurücklegen. Dabei wird in jeder einzelnen von $K$ Schichten Warners Methode eingesetzt. Als Gesamtschätzer $\widehat{\pi}_{A}^{K W}$ für $\pi_{A}$ ergibt sich die mit den relativen Schichtgrößen gewichtete Summe der Schätzer $\widehat{\pi}_{A, k}^{W}$ für die Schichtanteile $\pi_{A, k}$, $k=1, \ldots, K$.

J. M. Kim and Elam (2005) verknüpften die Theorie der geschichteten Zufallsauswahlen mit dem Befragungsdesign von Mangat and Singh (1990). Und auch deren Gesamtschätzer $\widehat{\pi}_{A}^{K E}$ für $\pi_{A}$ ergibt sich aus der mit den relativen Schichtgrößen gewichteten Summe der Schätzer $\widehat{\pi}_{A, k}^{M S}$ für die Schichtanteile $\pi_{A, k}$. Die Varianzen von $\widehat{\pi}_{A}^{K W}$ und $\widehat{\pi}_{A}^{K E}$ für Ziehen ohne und Ziehen mit Zurücklegen folgen der Theorie der geschichteten Zufallsauswahlen (siehe dazu etwa Quatember, 2001, S.49ff).

Als Beispiel für eine randomisierte Antworttechnik, die ein zusätzliches Designelement aufweist und sich nicht ausschließlich aus Warners bzw. Greenberg et al.'s Vorgehen ableitet, wird Mangat (1994) in unsere Untersuchung miteinbezogen. Seine zweistufige Strategie, deren konkreter Verlauf bei den einzelnen Befragten den Interviewern wiederum verborgen bleiben muss, verlangt vom Respondenten in jedem Falle eine ,jaAntwort", wenn er zur Gruppe $U_{A}$ gehört. Wenn dies nicht der Fall ist, dann ist Warners Befragungsdesign anzuwenden. Der für $\pi_{A}$ wiederum erwartungstreue Schätzer $\widehat{\pi}_{A}^{M 2}$ ist hierbei (ebd., S.94)

$$
\hat{\pi}_{A}^{M 2}=\frac{\hat{\pi}_{y}-1+p}{p} .
$$

Für Ziehen mit Zurücklegen gibt Mangat (1994) die Varianz dieses Schätzers an (vgl. ebd., S.94)

$$
V\left(\hat{\pi}_{A}^{M 2}\right)=\frac{\pi_{A}\left(1-\pi_{A}\right)}{n}+\frac{\left(1-\pi_{A}\right)(1-p)}{n p} .
$$

Bei Ziehen ohne Zurücklegen lässt sich die Schätzervarianz darstellen als

$$
V\left(\hat{\pi}_{A}^{M 2}\right)=\frac{\pi_{A}\left(1-\pi_{A}\right)}{n} \frac{N-n}{N-1}+\frac{\left(1-\pi_{A}\right)(1-p)}{n p} .
$$


Der Beweis von (10) befindet sich im Anhang. Die jeweils rechten Summanden der beiden Schätzervarianzen (9) und (10) entsprechen dem Streuungszuwachs, der durch das gewählte Befragungsdesign im Vergleich zu einer erfolgreich durchgeführten direkten Befragung entsteht.

\section{Die Simulationsstudie}

\subsection{Festlegung der Parameter}

Unter der Annahme, dass durch die verschiedenen (mehr oder weniger verschlungenen) Wege der oben beschriebenen randomisierten Antworttechniken die volle Kooperationsbereitschaft der Befragten gewährleistet wird, lassen sich diese Befragungsdesigns hinsichtlich ihrer Effizienz bei der Schätzung von $\pi_{A}$ direkt miteinander vergleichen. Um die tatsächlichen Schätzerverteilungen bei Ziehen ohne Zurücklegen auch visuell miteinander vergleichen zu können, wurde eine Simulationsstudie von Freudenthaler (2006) fortgeführt, in der bei Vorgabe der notwendigen Designparameter alle beschriebenen Schätzer in einer Million uneingeschränkt zufällig ohne Zurücklegen gezogenen Stichproben errechnet wurden. Die designspezifischen Parameter wurden nach den diesbezüglich vorgegebenen Richtlinien in den Originalaufsätzen bestimmt.

Die allgemeinen Parameter werden für die Studie folgendermaßen festgelegt: Die Größe der Grundgesamtheit sei $N=1.000$, jene der eine Million uneingeschränkt ohne Zurücklegen gezogenen Stichproben $n=100$ und der zu schätzende Parameter $\pi_{A}=0.2$ bzw. in einer 2. Simulation $\pi_{A}=0.1$. (Man stelle sich beispielsweise eine Großbetrieb vor und der interessierende Parameter sei der Anteil der Beschäftigten, die Betriebseigentum für private Zwecke entwenden.) Als Referenzstrategie dient die direkte Befragung in einer uneingeschränkten Zufallsstichprobe ohne Zurücklegen unter der (bei der nachgefragten Thematik eben nicht einzuhaltenden) Annahme von Fullresponse mit der Berechnung des unverzerrten Schätzers $\widehat{\pi}_{A}^{d i r}$ (in den nachfolgenden Boxplots und Tabellen als „dir“ bezeichnet). $\widehat{\pi}_{A}^{d i r}$ ist der Anteil an Elementen der Stichprobe, die zu $U_{A}$ gehören.

Für die Simulation von Warners Befragungsdesign zur Bestimmung der Verteilung des Schätzers $\widehat{\pi}_{A}^{W}$ (in Boxplots und Tabellen: „,W“) wird - in Einklang mit Greenberg et al.'s Überlegungen dazu - die Wahrscheinlichkeit $p=0.8$ gewählt (vgl. Greenberg et al., 1969, S.526).

Für Greenberg et al.'s Strategie zur Bestimmung des Schätzers $\widehat{\pi}_{A}^{G}$ bei bekanntem $\pi_{B}\left(,, G(B)^{\text {“ }}\right)$ und für diese Strategie anwendende Methoden sei zusätzlich zu $p=0.8$ entsprechend den von den Autoren angegebenen Regeln für die Wahl der Eigenschaft $B$ (bei $\pi_{A}<0.5$ ) $\pi_{B}=0.25$ bzw. 0.05. In unserem oben genannten Beispiel könnte etwa $\pi_{B}$ der Anteil jener Beschäftigten sein, die mit dem Privatauto in die Arbeit fahren.

Bei unbekanntem $\pi_{B}$ werden die zur Berechnung des Schätzers $\widehat{\pi}_{A}^{G^{\prime}}\left(, G^{\prime}(n 2, p 2, B){ }^{\text {“c }}\right)$ zusätzlich zu $p_{1}=0.8$ und $\pi_{B}$ benötigten Parameter folgendermaßen festgelegt: Die Aufteilung des Stichprobenumfanges $n$ auf die beiden Stichproben dieses Verfahrens erfolgt einmal gleichmäßig $\left(n_{1}=n_{2}=50\right)$ und einmal varianzoptimal (siehe Abschnitt 2.1). Als Wahrscheinlichkeit der heiklen Frage in der 2. Stichprobe wird $p_{2}=0.2$ (vgl. Greenberg et al., 1969, S.526 bzw. $p_{2}=0$ (vgl. Moors, 1971, S.627) gewählt. 
Bei den zweistufigen Verfahren aus Abschnitt 2.2 wird für die Berechnung von $\widehat{\pi}_{A}^{M S}$ $\left(, M S^{\prime \prime}\right)$ die Wahrscheinlichkeit $q$, schon auf der 1. Stufe nach der Zugehörigkeit zur Gesamtheit $U_{A}$ zu fragen, gleich $p$ gesetzt. Der Parameter $\pi_{B}$, der zur Berechnung von $\widehat{\pi}_{A}^{M 1}$ $\left(,, M 1(B)^{\star \prime}\right)$ zusätzlich benötigt wird, variiert wie oben beschrieben.

Für die Anwendung geschichteter Stichprobenverfahren mit proportionaler und optimaler Aufteilung des Stichprobenumfangs auf die Schichten zur Berechnung von $\widehat{\pi}_{A}^{K W}$

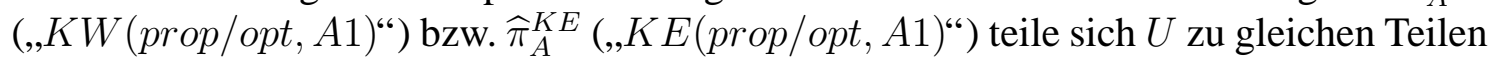
so auf zwei Schichten auf (in unserem „Betriebsbeispiel“ könnte man die Gesamtheit der im Betrieb beschäftigten Personen z.B. in weibliche und männliche Arbeitnehmer oder in Arbeiter und Angestellte zerlegen), dass für $\pi_{A}=0.2$ gilt: $\pi_{A, 1}=0.24$ (bzw. zur Erzielung eines höheren Schichtungseffektes $\pi_{A, 1}=0.35$ ) und $\pi_{A, 2}=0.16$ (bzw. 0.05). Für die Simulation mit $\pi_{A}=0.1$ gilt $\pi_{A, 1}=0.12$ (bzw. $\pi_{A, 1}=0.174$ ) und $\pi_{A, 2}=0.08$ (bzw. 0.026).

Schließlich wird noch die Verteilung des Schätzers $\widehat{\pi}_{A}^{M 2}$ (vgl. Mangat, 1994) mit der Wahrscheinlichkeit $p=0.8$ für die Auswahl der heiklen Frage, wenn das Stichprobenelement nicht die Eigenschaft $A$ besitzt, betrachtet, und in Boxplots und Tabellen mit , $M 2^{\text {“ }}$ bezeichnet.

\subsection{Die Simulationsergebnisse}

Die Verteilungen aller Schätzer können bei den gegebenen Parametern nur als annähernd normal mit einer durch den geringen Stichprobenumfang und der im Vergleich dazu auch geringen Größe der Grundgesamtheit bedingten und auch unterschiedlich ausgeprägten Schiefe betrachtet werden (siehe auch Freudenthaler, 2006, S.29). Die Boxplots der zu den verschiedenen randomisierten Antworttechniken gehörenden Stichprobenverteilungen der Schätzer sind in den Abbildungen 1 und 2 nach der Schätzervarianz gereiht. Die „Whiskers“ der Boxplots geben jeweils die Spannweite der 1 Million Ergebnisse an. Die Box erstreckt sich - wie üblich - vom unteren zum oberen Quartil und die senkrechte Linie in der Box kennzeichnet den Median der Verteilung.

Die Mittelwerte der allesamt erwartungstreuen Schätzer betragen über alle Simulationen (bei Rundung auf 4 Nachkommestellen) jeweils 0.2000 bzw. 0.1000. In den dazu gehörenden Tabellen 1 und 2 finden sich neben den simulierten Medianen und Varianzen auch die simulierten 95\%-Zufallsstreifen, die die mittleren 95\% der Schätzergebnisse enthalten und in denen sich die überwiegend vorherrschende Rechtsschiefe recht deutlich manifestiert.

An die Qualität der direkten Befragung kommt natürlich keines der betrachteten Befragungsdesigns heran. Nochmals sei jedoch betont, dass im Falle einer sensitiven Thematik bei direkter Befragung mit einer hohen Verweigerungs- und Falschantwortrate zu rechnen ist und die Fullresponseannahme somit unzutreffend ist. Erst die randomisierten Antworttechniken sollen wahrheitsgetreuen Fullresponse garantieren. Hinsichtlich der erzielbaren Schätzergenauigkeit unterscheiden sich die betrachteten unterschiedlichen Befragungsdesigns teilweise ganz erheblich voneinander. So sind Warners und Greenberg et al.'s (mit oder ohne gegebenem $\pi_{B}$ ) Techniken am ineffizientesten. Im Vergleich zur direkten Befragung weisen Sie einen bis zu mehr als doppelt so breiten 95\%-Zufallsstreifen auf. Unter diesen Basistechniken des randomisierten Antwortens ist natürlich Technik $G$ 


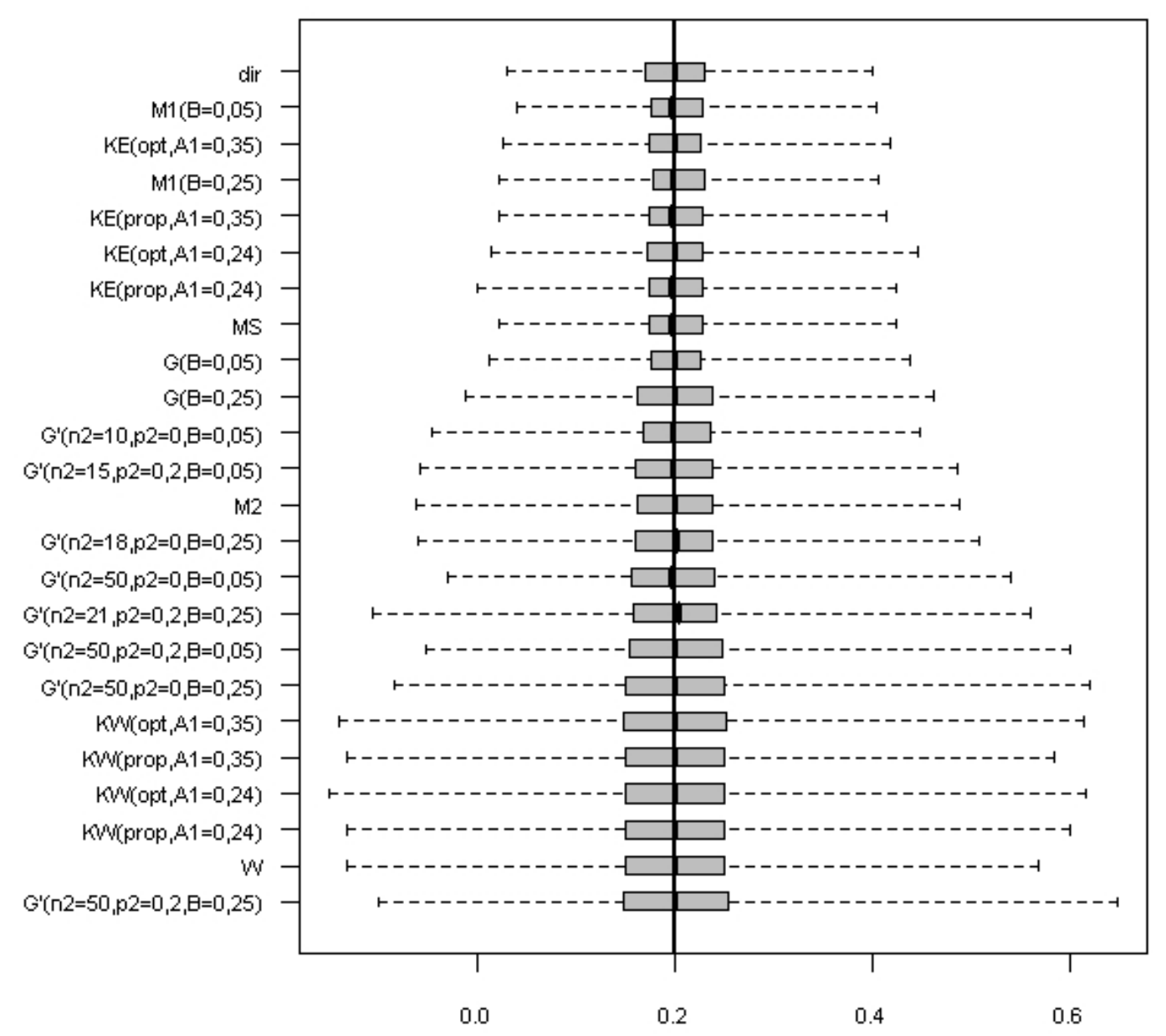

Abbildung 1: Box-Plots der Schätzer für $\pi_{A}=0.2$

(Greenberg et al. mit bekanntem $\pi_{B}$ ) die wirksamste, wobei für $G$ und $G^{\prime}$ (Greenberg et al. mit unbekanntem $\pi_{B}$ ) gleichermaßen gilt, dass die Schätzerstreuung mit $\pi_{B} \rightarrow 0$ abnimmt. $G^{\prime}$ liefert ferner eine höhere Schätzergenauigkeit, wenn $p_{2}=0$ gewählt wird, wie dies Moors (1971) vorgeschlagen hat.

Die geschichtete Anwendung von Warners Strategie $(K W)$ lässt im Vergleich zum ungeschichteten Befragungsdesign $W$ einen positiven Schichtungseffekt erkennen, dessen Ausmaß jedoch - sowohl bei proportionaler als auch bei optimaler Schichtung - eher moderat ausfällt. Am größten ist er bei optimaler Schichtung und bei größtem Unterschied der Anteile von $A$ zwischen den Schichten, wobei jedoch zu bedenken ist, dass für die Anwendung der optimalen Schichtung a priori-Schätzungen für die varianzrelevanten Parameter benötigt werden, deren Beschaffung aufwendiger sein kann als der dadurch erzielte Effekt.

Das Befragungsdesign M2 von Mangat (1994), bei dem Warners Technik nur dann zur Anwendung kommt, wenn die Erhebungseinheit nicht zu $U_{A}$ gehört, lässt bei den gegebenen Parametern eine Schätzung des Parameters $\pi_{A}$ zu, deren 95\%-Zufallsstreifen um ziemlich genau $1 / 4$ schmäler ausfällt als bei $W$ selbst. Das zweistufige Design $M S$, das vor der Anwendung von Warners Verfahren schon eine Stufe mit der Frage nach der Zugehörigkeit zu $U_{A}$ einfügt, lässt hier eine in etwa dem Verfahren $G$ entsprechen- 
Tabelle 1: Mediane, Schätzervarianzen (in $10^{-3}$ ) und 95\%-Zufallsstreifen bei $\pi_{A}=0.2$

\begin{tabular}{lccc}
\hline Schätzer & Median & Schätzervarianz & Zufallsstreifen \\
\hline $\operatorname{dir}$ & 0.2000 & 1.4410 & {$[0.1300 ; 0.2800]$} \\
$M 1(B=0.05)$ & 0.1958 & 1.5353 & {$[0.1229 ; 0.2791]$} \\
$K E($ opt,$A 1=0.35)$ & 0.1990 & 1.5754 & {$[0.1265 ; 0.2801]$} \\
$M 1(B=0.25)$ & 0.1979 & 1.5889 & {$[0.1250 ; 0.2813]$} \\
$K E($ prop,$A 1=0.35)$ & 0.1957 & 1.6919 & {$[0.1196 ; 0.2826]$} \\
$K E($ opt,$A 1=0.24)$ & 0.2002 & 1.8766 & {$[0.1169 ; 0.2875]$} \\
$K E($ prop,$A 1=0.24)$ & 0.1957 & 1.8794 & {$[0.1196 ; 0.2826]$} \\
$M S$ & 0.1957 & 1.8950 & {$[0.1196 ; 0.2826]$} \\
$G(B=0.05)$ & 0.2000 & 2.0399 & {$[0.1125 ; 0.2875]$} \\
$G(B=0.25)$ & 0.2000 & 2.4208 & {$[0.1125 ; 0.3000]$} \\
$G^{\prime}(n 2=10, p 2=0, B=0.05)$ & 0.1972 & 2.5847 & {$[0.1000 ; 0.3056]$} \\
$G^{\prime}(n 2=15, p 2=0.2, B=0.05)$ & 0.1974 & 3.3365 & {$[0.0902 ; 0.3132]$} \\
$M 2$ & 0.2000 & 3.4476 & {$[0.0875 ; 0.3125]$} \\
$G^{\prime}(n 2=18, p 2=0, B=0.25)$ & 0.2023 & 3.6497 & {$[0.0843 ; 0.3228]$} \\
$G^{\prime}(n 2=50, p 2=0, B=0.05)$ & 0.1950 & 4.3026 & {$[0.0800 ; 0.3350]$} \\
$G^{\prime}(n 2=21, p 2=0.2, B=0.25)$ & 0.2035 & 4.5410 & {$[0.0735 ; 0.3386]$} \\
$G^{\prime}(n 2=50, p 2=0.2, B=0.05)$ & 0.2000 & 5.0255 & {$[0.0667 ; 0.3467]$} \\
$G^{\prime}(n 2=50, p 2=0, B=0.25)$ & 0.2000 & 5.2561 & {$[0.0650 ; 0.3500]$} \\
$K W($ opt,$A 1=0.35)$ & 0.2001 & 5.6429 & {$[0.0558 ; 0.3497]$} \\
$K W($ prop,$A 1=0.35)$ & 0.2000 & 5.6883 & {$[0.0500 ; 0.3500]$} \\
$K W($ opt,$A 1=0.24)$ & 0.1989 & 5.8691 & {$[0.0498 ; 0.3499]$} \\
$K W($ prop,$A 1=0.24)$ & 0.2000 & 5.8773 & {$[0.0500 ; 0.3500]$} \\
$W$ & 0.2000 & 5.8822 & {$[0.0500 ; 0.3500]$} \\
$G^{\prime}(n 2=50, p 2=0.2, B=0.25)$ & 0.2000 & 6.1307 & {$[0.0533 ; 0.3600]$} \\
\hline & & &
\end{tabular}

de Schätzung von $\pi_{A}$ zu. Der zusätzliche Genauigkeitsgewinn von $M S$ bei Schichtung (Befragungsdesign $K E$ ) ist wiederum von geringem Ausmaß.

Sehr beeindruckend schneidet Methode $M 1$ ab. Diese zweistufige Anwendung der randomisierten Antworttechnik $G$ nach Mangat (1992) kommt der direkten Befragung am nächsten und ist (wie $G$ selbst) am effizientesten, wenn $\pi_{B} \rightarrow 0$ geht. Vorausgesetzt wird in Hinblick auf die praktische Relevanz dieses Ergebnisses - wie oben bereits erwähnt -, dass die Kooperationsbereitschaft der Respondenten durch die designbedingte „Verschleierung“ der Erhöhung der Gesamtwahrscheinlichkeit für das Stellen der Frage nach der Zugehörigkeit zur Gesamtheit $U_{A}$ durch die Zweistufigkeit tatsächlich erhalten bleibt.

Ein Vergleich der Ergebnisse in Hinblick auf die Auswirkung eines geringeren Anteils $\pi_{A}$ der Eigenschaft $A$ auf die Schätzer bringt keine gravierenden Veränderungen des Gesamtbildes (Abbildung 2 und Tabelle 2). Das Auffälligste an den Simulationen bei $\pi_{A}=0.1$ ist der Umstand, dass sich der Teil der in den negativen Wertebereich fallenden Spannweite der Schätzer für $\pi_{A}$ deutlich vergrößert. Dies ist jedoch von eher theoretischem Interesse. In der Praxis wird man in einem solchen Fall den Schätzer auf Null setzen und damit die Stichprobenverteilung an dieser Stelle stutzen (vgl. etwa Chaudhuri und Mukerjee, 1987, S.8f). 


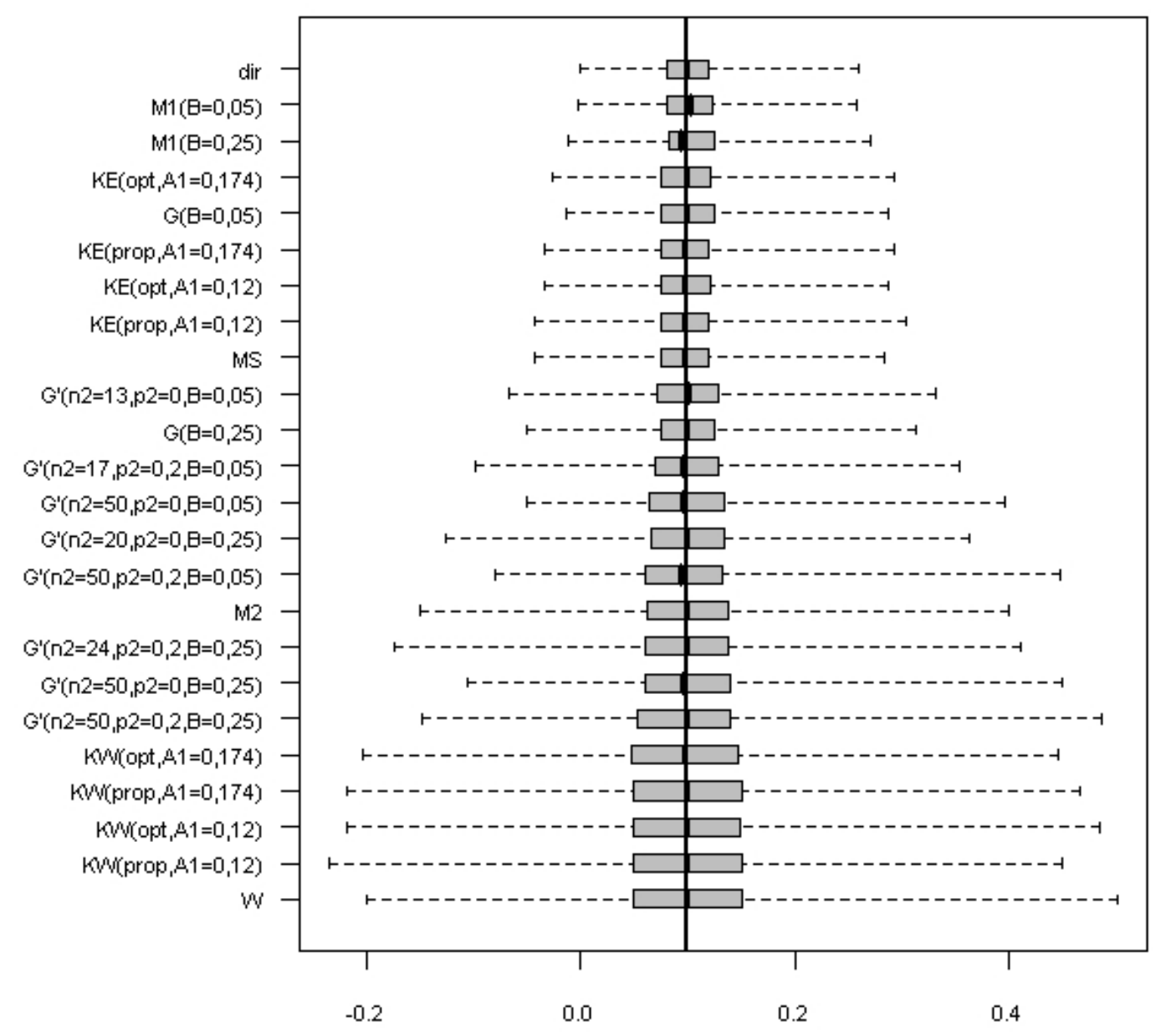

Abbildung 2: Box-Plots der Schätzer für $\pi_{A}=0.1$

\section{Zusammenfassung}

Randomisierte Antworttechniken bieten die Möglichkeit, durch Bewahrung der Privatsphäre der Respondenten Schätzungen für unbekannte Parameter sensitiver Merkmale zu ermöglichen, bei denen die Schätzung durch direkte Befragung wegen Antwortverweigerung und bewusste Falschbeantwortung beeinträchtigt wäre. Die durch eine cleveres Befragungsdesign gewährleistete Erhöhung der Privatsphäre wird durch einen Genauigkeitsverlust der Schätzung erkauft, der je nach Befragungsstrategie unterschiedlich hoch ausfallen kann.

Die Schätzervarianzen für Anteilschätzer sind auch bei Ziehen ohne Zurücklegen formal darstellbar. Allgemeine Aussagen über das Ausmaß der Erhöhung der Schätzervarianz im Vergleich verschiedener solcher Techniken sind jedoch wegen der Komplexität der (mehr oder weniger) frei wählbaren Designparameter nur bedingt möglich. In der Simulationsstudie wurden diese Parameter nach den Vorgaben aus den Originalaufsätzen festgelegt.

Es zeigt sich, dass sich die Basisdesigns nach Warner (1965) und Greenberg et al. (1969) durch Anwendung geschichteter Stichprobenverfahren effizienter gestalten lassen. Mehrstufige Vorgehensweisen und ergänzende Designelemente können die Effizienz sogar so deutlich erhöhen, dass eine Annäherung an die Qualität der Schätzung an jene 
Tabelle 2: Mediane, Schätzervarianzen (in $10^{-3}$ ) und 95\%-Zufallsstreifen bei $\pi_{A}=0.1$

\begin{tabular}{lccc}
\hline Schätzer & Median & Schätzervarianz & Zufallsstreifen \\
\hline $\operatorname{dir}$ & 0.1000 & 0.8110 & {$[0.0500 ; 0.1600]$} \\
$M 1(B=0.05)$ & 0.1021 & 0.8681 & {$[0.0500 ; 0.1646]$} \\
$M 1(B=0.25)$ & 0.0938 & 0.9400 & {$[0.0417 ; 0.1667]$} \\
$K E($ opt,$A 1=0.174)$ & 0.0991 & 1,1462 & {$[0.0377 ; 0.1692]$} \\
$G(B=0.05)$ & 0.1000 & 1,1848 & {$[0.0375 ; 0.1750]$} \\
$K E($ prop,$A 1=0.174)$ & 0.0978 & 1,2171 & {$[0.0326 ; 0.1739]$} \\
$K E($ opt,$A 1=0.12)$ & 0.0977 & 1,2554 & {$[0.0322 ; 0.1721]$} \\
$K E($ prop,$A 1=0.12)$ & 0.0978 & 1,2619 & {$[0.0326 ; 0.1739]$} \\
$M S$ & 0.0978 & 1,2653 & {$[0.0326 ; 0.1739]$} \\
$G^{\prime}(n 2=13, p 2=0, B=0.05)$ & 0.1006 & 1,6124 & {$[0.0239 ; 0.1819]$} \\
$G(B=0.25)$ & 0.1000 & 1,6649 & {$[0.0250 ; 0.1875]$} \\
$G^{\prime}(n 2=17, p 2=0.2, B=0.05)$ & 0.0964 & 2,0323 & {$[0.0125 ; 0.1928]$} \\
$G^{\prime}(n 2=50, p 2=0, B=0.05)$ & 0.0950 & 2,5300 & {$[0.0100 ; 0.2100]$} \\
$G^{\prime}(n 2=20, p 2=0, B=0.25)$ & 0.1000 & 2,7029 & {$[0.0031 ; 0.2063]$} \\
$G^{\prime}(n 2=50, p 2=0.2, B=0.05)$ & 0.0933 & 2,9546 & {$[0.0067 ; 0.2133]$} \\
$M 2$ & 0.1000 & 3,0560 & {$[0.0000 ; 0.2125]$} \\
$G^{\prime}(n 2=24, p 2=0.2, B=0.25)$ & 0.0987 & 3,3528 & {$[-0.0095 ; 0.2178]$} \\
$G^{\prime}(n 2=50, p 2=0, B=0.25)$ & 0.0950 & 3,6818 & {$[-0.0100 ; 0.2250]$} \\
$G^{\prime}(n 2=50, p 2=0.2, B=0.25)$ & 0.1000 & 4,3173 & {$[-0.0200 ; 0.2333]$} \\
$K W($ opt,$A 1=0.174)$ & 0.0976 & 5,1713 & {$[-0.0363 ; 0.2451]$} \\
$K W($ prop,$A 1=0.174)$ & 0.1000 & 5,1978 & {$[-0.0333 ; 0.2500]$} \\
$K W($ opt,$A 1=0.12)$ & 0.0995 & 5,2460 & {$[-0.0345 ; 0.2486]$} \\
$K W($ prop,$A 1=0.12)$ & 0.1000 & 5,2495 & {$[-0.0333 ; 0.2500]$} \\
$W$ & 0.1000 & 5,2558 & {$[-0.0333 ; 0.2500]$} \\
\hline
\end{tabular}

der direkten Befragung möglich erscheint. Diese Annäherung stößt jedoch dort an ihre Grenzen, wo durch die Festlegung der Designparameter der wesentliche Unterschied zur direkten Befragung, das ist die Kooperationsbereitschaft der Respondenten, verloren geht. Wo für die einzelnen Methoden diese Grenzen zu ziehen sind, lässt sich letztlich nur in der praktischen Umsetzung der Verfahren testen.

\section{Anhang}

\section{Beweis von (4)}

Es gilt

$$
V\left(\hat{\pi}_{A}^{G}\right)=\frac{1}{p^{2}} V\left(\hat{\pi}_{y}\right) .
$$


Es seien hinsichtlich der Erhebungseinheit $i$

$$
\begin{aligned}
& v_{i}=\left\{\begin{array}{l}
1 \text { wenn } i \text { die Eigenschaft } A \text { aufweist, } \\
0 \text { sonst, }
\end{array}\right. \\
& w_{i}=\left\{\begin{array}{l}
1 \text { wenn } i \text { die Eigenschaft } B \text { aufweist, } \\
0 \text { sonst, }
\end{array}\right. \\
& x_{i}=\left\{\begin{array}{l}
1 \text { wenn } i \text { die Frage nach der Mitgliedschaft in } U_{A} \text { erhält, } \\
0 \text { sonst, }
\end{array}\right. \\
& y_{i}=\left\{\begin{array}{l}
1 \text { wenn } i \text { mit }, j \mathrm{ja} \text { “ antwortet, } \\
0 \text { sonst. }
\end{array}\right.
\end{aligned}
$$

Ferner gilt

$$
y_{i}=v_{i} x_{i}+w_{i}\left(1-x_{i}\right)=v_{i} x_{i}+w_{i}-w_{i} x_{i} .
$$

Es ist $\widehat{\pi}_{y}=\sum_{s} y_{i} / n$ und somit wird (11) zu

$$
V\left(\hat{\pi}_{A}^{G}\right)=\frac{1}{n^{2} p^{2}} V\left(\sum_{s} y_{i}\right)
$$

Nun ist

$$
V\left(\sum_{s} y_{i}\right)=\mathrm{E}\left(\sum_{s} y_{i}^{2}\right)+\mathrm{E}\left(\sum_{s(i \neq j)} y_{i} y_{j}\right)-\mathrm{E}^{2}\left(\sum_{s} y_{i}\right) .
$$

Für Ziehen mit und ohne Zurücklegen gilt gleichermaßen

$$
\mathrm{E}\left(\sum_{s} y_{i}^{2}\right)=\mathrm{E}\left(\sum_{s} y_{i}\right)=n\left(p \pi_{A}+(1-p) \pi_{B}\right)
$$

Für den 2. Summanden in (13) gilt

$$
\begin{aligned}
\mathrm{E}\left(\sum_{s(i \neq j)} y_{i} y_{j}\right)= & n(n-1) \mathrm{E}\left(v_{i} v_{j} x_{i} x_{j}+w_{i} v_{j} x_{j}-w_{i} x_{i} v_{j} x_{j}+v_{i} x_{i} w_{j}\right. \\
& \left.+w_{i} w_{j}-w_{i} w_{j} x_{i}-v_{i} x_{i} w_{j} x_{j}--w_{i} w_{j} x_{j}+w_{i} w_{j} x_{i} x_{j}\right)
\end{aligned}
$$

Es ist

$$
\mathrm{E}\left(v_{i} v_{j}\right)= \begin{cases}\pi_{A}^{2} & \text { bei Ziehen mit Zurücklegen, } \\ \frac{\pi_{A}\left(N \pi_{A}-1\right)}{N-1} \text { bei Ziehen ohne Zurücklegen }\end{cases}
$$

und

$$
\mathrm{E}\left(w_{i} w_{j}\right)= \begin{cases}\pi_{B}^{2} & \text { bei Ziehen mit Zurücklegen, } \\ \frac{\pi_{B}\left(N \pi_{B}-1\right)}{N-1} \text { bei Ziehen ohne Zurücklegen. }\end{cases}
$$

Somit ergibt sich für Ziehen mit Zurücklegen

$$
\mathrm{E}\left(\sum_{s(i \neq j)} y_{i} y_{j}\right)=n(n-1)\left(\pi_{A}^{2} p^{2}+2 \pi_{A} \pi_{B} p-2 \pi_{A} \pi_{B} p^{2}+\pi_{B}^{2}-2 \pi_{B}^{2} p+\pi_{B}^{2} p^{2}\right)
$$


und für Ziehen ohne Zurücklegen

$$
\begin{aligned}
\mathrm{E}\left(\sum_{s(i \neq j)} y_{i} y_{j}\right)= & n(n-1)\left(\frac{\pi_{A}\left(N \pi_{A}-1\right)}{N-1} p^{2}+2 \pi_{A} \pi_{B} p-2 \pi_{A} \pi_{B} p^{2}+\right. \\
& \left.+\frac{\pi_{B}\left(N \pi_{B}-1\right)}{N-1}-2 \frac{\pi_{B}\left(N \pi_{B}-1\right)}{N-1} p+\frac{\pi_{B}\left(N \pi_{B}-1\right)}{N-1} p^{2}\right) .
\end{aligned}
$$

Der Subtrahend auf der rechten Seite von (13) ist bei Ziehen mit wie auch bei Ziehen ohne Zurücklegen folgendermaßen darstellbar

$$
\mathrm{E}^{2}\left(\sum_{s} y_{i}\right)=n^{2}\left(\pi_{A}^{2} p^{2}+2 \pi_{A} \pi_{B} p-2 \pi_{A} \pi_{B} p^{2}+\pi_{B}^{2}-2 \pi_{B}^{2} p+\pi_{B}^{2} p^{2}\right) .
$$

Für Ziehen mit Zurücklegen ergibt sich für (12) aus (13) bis (15) und (17) Ergebnis (6).

Für Ziehen ohne Zurücklegen ergibt sich auf Grund der beim 2. Summanden (16) auftretenden Abweichung im Vergleich zu Ziehen mit Zurücklegen ausgehend von (12) folgende Varianzdarstellung

$$
\begin{aligned}
V\left(\hat{\pi}_{A}^{G}\right)= & \frac{\pi_{y}\left(1-\pi_{y}\right)}{n p^{2}}-\frac{1}{n^{2} p^{2}}\left[\frac{n(n-1)}{N-1} p^{2} \pi_{A}\left(1-\pi_{A}\right)+\frac{n(n-1)}{N-1} \pi_{B}\left(1-\pi_{B}\right)-\right. \\
& \left.-2 \frac{n(n-1)}{N-1} p \pi_{B}\left(1-\pi_{B}\right)++\frac{n(n-1)}{N-1} p^{2} \pi_{B}\left(1-\pi_{B}\right)\right] .
\end{aligned}
$$

Daraus ergibt sich endlich (7)

$$
\begin{aligned}
V\left(\hat{\pi}_{A}^{G}\right) & =\frac{\pi_{y}\left(1-\pi_{y}\right)}{n p^{2}}-\frac{n-1}{n(N-1)}\left[\pi_{A}\left(1-\pi_{A}\right)+\pi_{B}\left(1-\pi_{B}\right)\left(\frac{1}{p^{2}}-\frac{2}{p}+1\right)\right] \\
& =\frac{\pi_{y}\left(1-\pi_{y}\right)}{n p^{2}}-\frac{n-1}{n(N-1)}\left[\pi_{A}\left(1-\pi_{A}\right)+\left(\frac{1-p}{p}\right)^{2} \pi_{B}\left(1-\pi_{B}\right)\right] .
\end{aligned}
$$

\section{Beweis von (10):}

Es seien $v_{i}$ und $y_{i}$ wie in Abschnitt (5.1) definiert. Für $x_{i}$ gilt

$$
x_{i}=\left\{\begin{array}{l}
1 \text { wenn } i \text { die Frage nach der Mitgliedschaft in } U_{A} \text { erhält, gegeben } i \in U_{A} \\
0 \text { sonst. }
\end{array}\right.
$$

Es gilt ferner

$$
y_{i}=v_{i}+\left(1-v_{i}\right)\left(1-x_{i}\right)=1-x_{i}+v_{i} x_{i} .
$$

Für den 1. Summanden in (13) ergibt sich

$$
\mathrm{E}\left(\sum_{s} y_{i}\right)=n\left(\pi_{A}+\left(1-\pi_{A}\right)(1-p)\right) .
$$

Der 2. Summand in (13) wird zu

$\mathrm{E}\left(\sum_{s(i \neq j)} y_{i} y_{j}\right)=n(n-1) \mathrm{E}\left(1-x_{i}+v_{i} x_{i}-x_{j}+x_{i} x_{j}-v_{i} x_{i} x_{j}+v_{j} x_{j}-v_{j} x_{i} x_{j}+v_{i} v_{j} x_{i} x_{j}\right)$. 
Dies ergibt für Ziehen mit Zurücklegen

$$
\mathrm{E}\left(\sum_{s(i \neq j)} y_{i} y_{j}\right)=n(n-1)\left(1-2 p+2 \pi_{A} p+p^{2}-2 \pi_{A} p^{2}+\pi_{A}^{2} p^{2}\right) .
$$

Für Ziehen ohne Zurücklegen ergibt sich hinsichtlich des 2. Summanden aus (13)

$$
\mathrm{E}\left(\sum_{s(i \neq j)} y_{i} y_{j}\right)=n(n-1)\left(1-2 p+2 \pi_{A} p+p^{2}-2 \pi_{A} p^{2}+\frac{\pi_{A}\left(N \pi_{A}-1\right)}{N-1} p^{2}\right)
$$

Der Subtrahend in (13) ist für das Befragungsdesign nach Mangat (1994) gegeben durch

$$
\mathrm{E}^{2}\left(\sum_{s} y_{i}\right)=n^{2}\left(1+2 \pi_{A} p-2 p+\pi_{A}^{2} p^{2}-2 \pi_{A} p^{2}+p^{2}\right)
$$

Für Ziehen mit Zurücklegen ergibt sich für (12) aus (13), (18), (19) und (21) das Ergebnis (9).

Für Ziehen ohne Zurücklegen ergibt sich auf Grund der beim 2. Summanden (20) auftretenden Abweichung im Vergleich zu Ziehen mit Zurücklegen ausgehend von (12) folgende Varianzdarstellung

$$
\begin{aligned}
V\left(\hat{\pi}_{A}^{M 2}\right) & =\frac{1}{n p^{2}}\left(n \frac{N-n}{N-1} p^{2} \pi_{A}\left(1-\pi_{A}\right)+n p-n p \pi_{A}-n p^{2}+n p^{2} \pi_{A}\right) \\
& =\frac{\pi_{A}\left(1-\pi_{A}\right)}{n} \frac{N-n}{N-1}+\frac{\left(1-\pi_{A}\right)(1-p)}{n p} .
\end{aligned}
$$

Und dies entspricht (10).

\section{Literatur}

Chaudhuri, A., und Mukerjee, R. (1987). Randomized Response - Theory and Techniques. New York: Marcel Dekker.

Freudenthaler, C. (2006). Randomisierte Response Techniken [Bachelorarbeit Betreuer: Andreas Quatember]. (Institut für Angewandte Statistik der Johannes Kepler Universität Linz)

Greenberg, B. G., Abul-Ela, A., Simmons, W. R., und Horvitz, D. G. (1969). The unrelated question randomized response model: Theoretical framework. Journal of the American Statistical Association, 64, 520-539.

Horvitz, D. G., Shah, B. V., und Simmons, W. R. (1967). The unrelated question randomized response model. 1967 Social Statistics Section Proceedings of the American Statistical Association, 65-72.

Kim, J.-I., und Flueck, J. A. (1978). Modifications of the Randomized Response Technique for Sampling Without Replacement. Proceedings of the Section on Survey Research Methods, American Statistical Association, 346-350. 
Kim, J. M., und Elam, M. E. (2005). A two-stage stratified Warner's randomized response model using optimal allocation. Metrika, 61, 1-7.

Kim, J. M., und Warde, W. D. (2004). A stratified Warner's randomized response model. Journal of Statistical Planning and Inference, 120, 155-165.

Mangat, N. S. (1992). Two Stage Randomized Response Sampling Procedure Using Unrelated question. Journal of the Indian Society of Agricultural Statistics, 44, 82-87.

Mangat, N. S. (1994). An Improved Randomized Response Strategy. Journal of the Royal Statistical Society, Series B, 56, 93-95.

Mangat, N. S., und Singh, R. (1990). An alternative randomized response procedure. Biometrika, 77, 439-442.

Moors, J. J. A. (1971). Optimization of the Unrelated Randomized Response Model. Journal of the American Statistical Association, 66, 627-629.

Quatember, A. (2001). Die Quotenverfahren - Stichprobentheorie und -praxis. Aachen: Shaker Verlag.

Warner, S. L. (1965). Randomized response: A survey technique for eliminating evasive answer bias. Journal of the American Statistical Association, 60, 63-69.

Adresse der Autoren:

Andreas Quatember und Christoph Freudenthaler

Johannes Kepler Universität Linz

IFAS-Institut für Angewandte Statistik

Abteilung für Datengewinnung und Datenqualität

Altenbergerstraße 69

A-4040 Linz, Österreich

E-mail: andreas .quatember@jku . at 